\title{
Étude de la réponse d'une interface air-eau à des mouvements tourbillonnaires dans l'air
}

\author{
On the response of an air-water interface \\ to vortex motion in the air flow
}

\author{
J.P. Giovanangeli, A. Ramamonjiarisoa, A. Memponteil \\ Institut de mécanique statistique de la turbulence, Marseille \\ R. Gelci, J.Y. Hervouet \\ Etablissement d'études et de recherches météorologiques, Brest
}

Les progrès réalisés au cours des dernières décennies sur la connaissance des mécanismes de génération et d'évolution des vagues de vent ont concerné plus particulièrement le comportement intrinsèque des champs de vagues [voir les travaux de Hasselmann (1968), Longuet-Higgins (1977), Mollo-Christensen et Ramamonjiarisoa (1978), etc.]. En effet, la connaissance des mécanismes de transfert d'énergie du vent aux vagues ne s'est pas améliorée d'une manière très significative, tant sur le plan théorique que sur le plan expérimental. Les expériences ont principalement montré les limitations des principaux modèles théoriques (Phillips, 1957; Miles, 1960) qui servent toujours de référence dans ce domaine. Un des obstacles majeurs réside dans la difficulté, en premier lieu, de décrire en détail la structure turbulente de l'écoulement d'air au-dessus de la surface et, en second lieu, d'analyser l'interaction entre les deux milieux fluides.

Il existe des raisons de penser que, au cours de la phase de génération des vagues par le vent, le mécanisme de résonance entre l'interface et les fluctuations de pression dans l'air joue un rôle prépondérant (Stoker, 1957; Phillips, 1957).

Une meilleure connaissance des processus de génération de ces vagues revêt une importance particulière pour la compréhension des interactions entre l'atmosphère et l'océan et pour une meilleure prévision de l'état de la mer.

En effet, l'évolution d'un champ de vagues est modélisée par l'équation de transfert radiatif :

$$
\begin{aligned}
& \frac{\partial F}{\partial t}(n, \theta, x, t)+C g \nabla F(n, \theta, x, t) \\
&= \alpha+\beta F+\gamma F+\mu F \\
& \text { I II III IV }
\end{aligned}
$$

où $F$ est la densité spectro-angulaire d'énergie des vagues et $C g$ sa vitesse de groupe. Les termes "sources" repré- sentent : I, les transferts linéaires de l'air à l'interface pris en compte, notamment dans la théorie de Phillips (1957); II : un terme d'interaction linéaire entre les deux milieux (théorie de Miles, 1957); III : interaction non linéaire entre différentes composantes spectrales (Hasselmann, 1962; Longuet-Higgins, 1962; Longuet-Higgins et Phillips, 1962); et IV: un terme de dissipation par viscosité.

Plus récemment, Gelci (1982) a développé un modèle numérique permettant de donner l'évolution d'une interface air-eau soumise à l'interaction d'une allée de tourbillons aériens mobiles convectés au-dessus de la surface. Les résultats sont tout à fait en accord avec ceux des modèles cités en ce qui concerne les ondes résonnantes directes et tant que la cambrure des vagues reste faible. Le modèle permet en outre d'étudier le comportement des ondes non-résonnantes qui pourraient notamment être représentées comme la superposition d’ondes forcées, d'ondes libres directes et d'ondes libres rétrogrades. Le modèle permet enfin d'étudier l'évolution des caractéristiques dynamiques et cinématiques des vagues ayant des cambrures élevées.

Le but de cet article est de présenter diverses études numériques théoriques et expérimentales menées conjointement par l'Institut de mécanique statistique de la turbulence et l'Etablissement d'études et de recherches météorologiques sur la réponse d'une interface air-eau soumise à l'interaction directe d'un champ de perturbations dans l'écoulement d'air (Gelci, 1982; Gelci, Ramamonjiarisoa et Hervouet, 1982; Giovanangeli et Memponteil, 1983; Memponteil, 1983). Divers résultats acquis sont rapportés et discutés.

Les études devraient, par une meilleure connaissance du processus de génération des vagues de vent par résonance, conduire ainsi à une modélisation du premier coefficient de l'équation de transfert radiatif. 


\section{Modèles de génération} des vagues de vent par résonance

La théorie de Phillips (1957) reste encore actuellement à la base des études sur la génération des vagues de vent. Elle étudie la réponse de l'interface air-eau à un champ de pression aléatoire dans l'écoulement d'air.

La cambrure des vagues est supposée assez faible pour permettre une linéarisation des équations de base écrites dans un repère se déplaçant horizontalement à la vitesse $U$ égale à la célérité des fluctuations de pression :
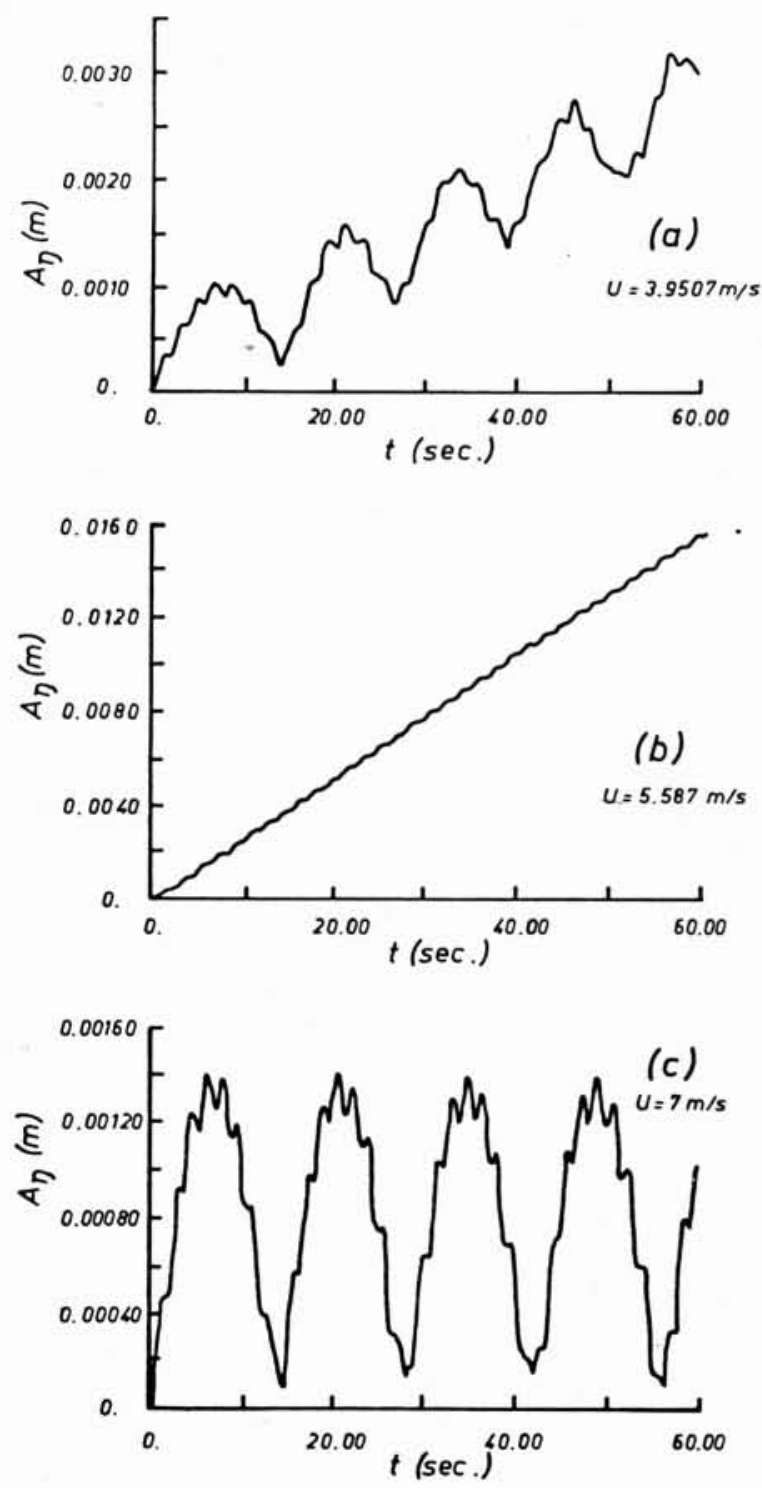

Figure 1 - Amplitude $A_{\eta}$ des fluctuations de l'interface $\eta(t)$ donnée par le modèle GEVA pour :

a) $U=3.9507 \mathrm{~m} / \mathrm{s}$

b) $U=5.587 \mathrm{~m} / \mathrm{s}$

c) $U=7 \mathrm{~m} / \mathrm{s}$
- l'équation de Bernoulli :

$\frac{p_{a}}{\rho_{e}}=\left(\frac{\partial \varphi}{\partial t}-U_{i} \frac{\partial \varphi}{\partial x}\right)_{2=0}-g \eta+\frac{\tau}{\rho_{e}}\left(\frac{\partial^{2} \eta}{\partial x_{1}^{2}}+\frac{\partial^{2} h}{\partial x_{2}^{2}}\right)$

- l'équation cinématique à l'interface :

$$
\left(\frac{\partial}{\partial t}-U_{i} \frac{\partial}{\partial x_{i}}\right) \eta=\left(\frac{\partial \varphi}{\partial z}\right)_{i=\eta}
$$

Le mouvement dans l'eau est supposé irrotationnel non visqueux et incompressible. Le potentiel des vitesses $\Phi$ dans l'écoulement d'eau vérifie l'équation de Laplace :

$$
\Delta \Phi=0
$$

Les perturbations sont supposées s'annuler en profondeur :

$$
\Phi \rightarrow 0 \text { pour } z \rightarrow-\infty
$$

$\eta$ est le niveau de l'interface, $x_{1}$ et $x_{2}$ les coordonnées horizontales, $z$ la coordonnée verticale, $p_{a}$ la fluctuation de pression dans l'air, $\rho$ e la densité de l'eau, $\tau$ la tension de surface à l'interface et $g$ l'accélération de sa pesanteur.

Les champs de pression et de vagues étant supposés homogènes, $p_{a}$ et $\eta$ peuvent être représentés dans l'espace des vecteurs d'onde par une intégrale de Fourier-Stieljes :

$$
\begin{aligned}
& \eta(\underline{x}, t)=\int \mathrm{d} A(\underline{k}, t) \mathrm{e}^{t \underline{k} x} \\
& p_{a}(\underline{x}, t)=\int \mathrm{d} \bar{\omega}(\underline{k}, t) \mathrm{e}^{\underline{k}-\underline{x}}
\end{aligned}
$$

Les équations (1), (2), (3), (4) et (5) permettent d'obtenir l'équation suivante :

$\mathrm{d} A^{\prime \prime}(\underline{k}, t)-2 i n, \mathrm{~d} A^{\prime}(\underline{k}, t)$

$-\left(n_{1}^{2}-n_{2}^{2}\right) \mathrm{d} A(\underline{k}, t)=\frac{-|\underline{k}|}{\rho_{e}} \mathrm{~d} \bar{\omega}(\underline{k}, t)$

avec :

$n_{1}=\underline{k} \cdot \underline{U}=|\underline{k}| \cdot|\underline{U}| \cdot \cos \alpha$

$n_{2}=\left(g|\underline{k}|+\tau \frac{|\underline{k}|^{3}}{\rho_{e}}\right)^{1 / 2}$

Cette équation de base relie les composantes harmoniques des fluctuations de pression à celles des mouvements de l'interface dans un repère se déplaçant à la célérité $U$.

Si on limite le temps $t$ de telle façon que $\mathrm{d} \bar{\omega}(\underline{k}, t)$ soit constant, Phillips montre que pour des ondes résonnantes $\left(n_{1} \simeq n_{2}\right)$ la densité spectrale du champ de vagues $\psi(\underline{k}, t)$ est liée à celle $\pi(\underline{k})$ du champ de pression par la relation :

$$
\psi(\underline{k}, t)=\frac{|\underline{k}|^{2} \pi(\underline{k}) t^{2}}{2 \rho_{e}^{2} n_{2}^{2}} \frac{1-\cos \chi}{\chi^{2}}
$$

$\chi=t\left\{|\underline{k}| U \cos \alpha-\left(g|\underline{k}|+\tau \frac{|\underline{k}|^{3}}{\rho_{e}}\right)^{1 / 2}\right\}$

et que :

$$
\overline{\eta^{2}} \simeq \frac{\overrightarrow{\mathrm{p}_{a}^{2}} \cdot t}{2 \sqrt{2} \cdot \rho_{e}^{2} \cdot U \cdot g}
$$


Comme indiqué dans l'introduction, la principale difficulté pour modéliser le transfert d'énergie du vent aux vagues provient de la complexité de la structure de l'écoulement d'air. Cependant celui-ci peut être décrit qualitativement comme une combinaison aléatoire de tourbillons ayant des caractéristiques cinématiques, dynamiques et géométriques diverses. Le modèle numérique GEVA (Gelci, 1982) est une étude de l'action du vent sur l'eau en modélisant l'écoulement d'air par une allée infinie de tourbillons advectés par un vent moyen au-dessus de l'interface.

La méthode employée est celle des singularités tourbillonnaires (Gelci, 1974; Gelci et Hervouet, 1981). On considère une allée horizontale de tourbillons ponctuels équidistants de $L$, située à l'altitude $d$ au-dessus de l'interface et convectée à la vitesse $U$. Les détails de calcul sont exposés dans l'article "Génération des vagues de gravité par des allées de tourbillons aériens mobiles" (Gelci, Ramamonjiarisoa et Hervouet, 1982).

Les principaux résultats obtenus font l'objet d'une analyse harmonique permettant de calculer l'amplitude et la célérité des composantes harmoniques se propageant à une célérité supérieure à $U$ (ondes directes) et celle d'ondes ayant une célérité pouvant être inférieure à $U$, et même négative (ondes rétrogrades).

Les premiers essais ont permis d'étudier les effets de la vitesse d'advection $U$ de l'allée de tourbillons pour un $L$ donné.

La figure 1 présente les résultats obtenus pour les valeurs suivantes :

$$
\begin{aligned}
L & =20 \mathrm{~m} \text { correspondant à } C(L)=5,587 \mathrm{~m} / \mathrm{s} \\
U & =3,95 \mathrm{~m} / \mathrm{s}(U<C(L)) \\
U & =7 \mathrm{~m} / \mathrm{s} \quad(U>C(L)) \\
U & =5,587 \mathrm{~m} / \mathrm{s}(U=C(L))
\end{aligned}
$$

$C$ étant la célérité de propagation hydrodynamique de l'onde de longueur d'onde $L$.

On constate que pour $U=C(L)$ l'amplitude de l'onde croît linéairement avec le temps. Pour $U \neq C$, elle décrit des oscillations dans le temps de période $L /(U-C(L))$.

Ces résultats sont en accord avec ceux obtenus par Phillips, notamment dans le cas où la condition de résonance est satisfaite. Bien que Phillips n'ait pas porté une attention particulière au cas d'ondes non résonnantes, on peut montrer (Giovanangeli et Memponteil, 1983), à partir des résultats de l'analyse de Phillips, l'existence des composantes " directes " et " rétrogrades " introduites par Gelci.

On peut montrer (Giovanangeli et Memponteil, 1983), à partir des équations de base linéarisées, que la réponse de l'interface soumise à une onde de pression bidimensionnelle du type $p=\mathrm{He}^{i(\omega t-k x)}$ se propageant à la célérité $W=\omega / k$ peut être décrite, comme dans l'analyse de Phillips, par l'équation :

$$
\frac{\mathrm{d}^{2} \eta}{\mathrm{d} t^{2}}+\alpha^{2} \eta \cong A \mathrm{e}^{\mathrm{k} o t}
$$

où $\tilde{\eta}$ est l'amplitude de $\eta=\tilde{\eta}(t) \mathrm{e}^{-i k x}$ (en supposant $\eta$ harmonique en $x$ ); avec

$$
A=\frac{k H}{\rho_{e}} \text { et } \alpha^{2}=\left(g k+\frac{\tau}{\rho_{e}} k^{3}\right)
$$

$\alpha$ étant la pulsation de l'onde libre $(A=0)$.
La recherche des solutions de l'équation (10) peut être effectuée de manière classique en utilisant la transformée de Laplace (Giovanangeli et Memponteil, 1983).

Si $\alpha=\omega$ (condition de résonance), on montre que l'interface est décrite par l'expression :

$$
\begin{aligned}
\eta=-\frac{\mathrm{kH}}{2 \rho_{e} \omega^{2}} & \cos k x \cos \omega t \\
& \quad-\frac{k H}{2 \rho_{e} \omega} \cdot t \cdot \sin (\omega t-k x)
\end{aligned}
$$

où le premier terme représente une onde stationnaire et le second terme une onde se propageant à la célérité $C=\omega / k=W$ et d'amplitude croissant linéairement au cours du temps. L'onde générée est bien une "onde résonnante "

Si $\alpha \neq \omega$ on montre, en utilisant la même méthode, que $\eta$ s'écrit :

$$
\begin{aligned}
\eta=\frac{k H}{2 \rho_{e} \alpha} \frac{1}{\alpha-\omega} & \cos (k x-\alpha t) \\
& +\frac{k H}{2 \rho_{e} \alpha} \frac{1}{\alpha+\omega} \cos (k x+\alpha t) \\
& \quad-\frac{k H}{\rho_{e}} \frac{1}{\alpha^{2}-\omega^{2}} \cos (k x-\omega t)
\end{aligned}
$$

$\mathrm{Si}$, de plus, $\alpha>\omega$, l'équation (12) se réduit à :

$$
\begin{aligned}
& \eta=\frac{k H}{2 \rho_{e}\left(\omega^{2}-\alpha^{2}\right)}\left\{\sin \left(\frac{\omega-\alpha}{2}\right) t\right\} \\
& \cdot\left\{\sin \left(k x-\left(\frac{\alpha+\omega}{2}\right) t\right)\right\} \\
& +\frac{k H}{2 \rho_{e}\left(\omega^{2}-\alpha^{2}\right)}\left\{\sin \left(\frac{\omega+\alpha}{2}\right) t\right\} \\
& \cdot\left\{\sin \left(k x+\left(\frac{\alpha-\omega}{2}\right) t\right)\right\}(13)
\end{aligned}
$$

Le premier membre de (13) représente une onde se propageant dans le sens du vent à la célérité apparente $(C+W) / 2$ et ayant une amplitude modulée dans le temps à la période $2 L /(C-W)$ soit $L /(C-W)$ pour le module de l'amplitude.

Le second terme représente une onde se propageant dans le sens du vent (ou même en sens contraire) à la célérité apparente $(W-C) / 2$ et ayant une amplitude modulée dans le temps à la période $2 L(C+W)$.

Ces résultats semblent en accord avec ceux de Gelci (1981), qui propose de décrire l'interface comme la superposition d'une onde "directe" et une onde "rétro " lorsque la condition de résonance n'est pas satisfaite. Gelci donne également les valeurs des célérités de phase et de périodes de modulation d'amplitude qui sont accord avec celles données ici. Les résultats concordent également lorsque la condition de résonance est satisfaite.

Enfin, l'équation (12) suggère que, pour des conditions de non résonance, le champ de vagues pourrait être décrit par la superposition de trois ondes, à savoir : une composante " libre " directe se propageant à la célérité hydrodynamique $+C$, une composante "rétrograde" se propageant à la célérité hydrodynamique $-C$ et une onde « forcée, c'est-à-dire directement liée à l'effet du vent, se propageant à la célérité $+W$. 
La combinaison de ces trois composantes permet de retrouver la formule (13).

Ces résultats sont également en accord avec les dernières analyses numériques effectuées par Gelci (1983).

Bien que le modèle numérique n'utilise pas explicitement la pression à l'interface, il a été mentionné que celle-ci est d'une importance capitale pour la description du processus de génération des vagues par le vent. Le modèle permet, entre autre, d'estimer les fluctuations de pression à l'interface associées à une allée de tourbillons mobiles au dessus d'une surface rigide ou déformable.

Dans le cas d'une surface rigide, l'expression de la pression à l'interface est donnée par :

$$
\begin{aligned}
p=P_{0}-2 \rho_{a}\left[-\frac{\Gamma W}{2 L} \frac{\text { th } \frac{\pi d}{L}}{1-\frac{\cos ^{2} \frac{\pi}{L}\left(x-\xi_{0}-W t\right)}{\operatorname{ch}^{2} \frac{\pi d}{L}}}\right. \\
+\frac{\Gamma^{2}}{4 L}\left(\frac{\operatorname{th} \frac{\pi d}{L}}{\left.\left.1-\frac{\cos ^{2} \frac{\pi}{L}\left(x-\xi_{0}-W t\right)}{\operatorname{ch}^{2} \frac{\pi d}{L}}\right)\right]}\right.
\end{aligned}
$$

où $\Gamma$ est l'intensité de chaque tourbillon aérien, $L$ la distance entre deux tourbillons consécutifs de l'allée, $\rho_{\mathrm{a}}$ la densité de l'air, $\boldsymbol{W}$ la vitesse de convection de l'allée située à l'altitude $d$ de la surface, $\xi_{0}$ l'abscisse du point central.

Dans le cas d'une interface libre, l'expression de la pression à l'interface est plus complexe :

$$
p=p_{0}+\frac{\rho_{a} \rho_{e}}{\bar{\rho}}\left(-g y-\frac{1}{8} G^{2}-\int_{0}^{s} J s \mathrm{ds}\right)
$$

où $\rho_{e}$ est la densité de l'eau, $\bar{\rho}=\left(\rho_{a}+\rho_{e}\right) / 2 ; y$, le niveau de l'interface; $g$, l'accélération de la pesanteur; $G$, la discontinuité de vitesse à l'interface et $J s$, la composante tangentielle, selon la surface libre, de l'accélération d'une particule. Le modèle permet également de relier l'amplitude $a_{n}$ de l'onde " forcée ", liée au champ de pression, à la composante $a_{p}$ de la pression :

$$
a_{\eta j} \simeq \frac{a_{p}}{\rho_{e} g} \frac{C^{2}}{W^{2}-C^{2}}
$$

Une expérience a été effectuée en soufflerie afin de contrôler les différentes hypothèses et résultats obtenus à partir des modèles théoriques et numériques (Memponteil, 1983; Giovanangeli et Memponteil, 1983).

Il a été possible d'étudier le comportement d'une interface air-eau soumise à l'action directe d'une distribution de tourbillons dans l'écoulement d'air au-dessus de la surface et convectée à des vitesses différentes ou voisines de celle correspondant à la condition de résonance.

\section{Dispositif expérimental et traitement des données}

Nous avons vu que la résonance de l'interface sous l'effet du champ de pression requiert la condition suivantes:

$$
W=C(k)
$$

où $W$ est la célérité de la composante harmonique du vecteur d'onde $k$ du champ de pression, et $C(k)$ la célérité théorique d'une onde de surface de même vecteur d'onde.

Si cette condition peut être satisfaite en site naturel (Giovanangeli, Revault d'Allonnes et Ramamonjiarisoa, 1978) par contre elle ne peut être remplie en soufflerie pour des vagues naturelles. En effet, l'expérience montre (Choï, 1977) que les premières vagues (de capillaritégravité) sont générées par des vitesses de l'écoulement d'air de l'ordre de $5 \mathrm{~m} / \mathrm{s}$ alors que les vagues se propagent à des célérités de l'ordre de $30 \mathrm{~cm} / \mathrm{s}$.

Pour satisfaire la condition de résonance en soufflerie, il est donc nécessaire de créer artificiellement un champ de perturbations dans l'écoulement d'air.

Un dispositif générateur de tourbillons dans l'écoulement d'air a été mis en œuvre. A ces tourbillons sont associés un champ de vitesse et de pression dont les caractéristiques cinématiques et dynamiques ont été ajustées de manière à pouvoir générer des ondes de surface dans des conditions d'observations pouvant satisfaire ou non la condition de résonance.

\subsection{Dispositif expérimental}

Les expériences ont été effectuées dans la maquette à l'échelle $1 / 5$ de la grande soufflerie de l'I.M.S.T. décrite en détail par Favre et Coantic (1974). Compte tenu des objectifs à atteindre, la veine aérodynamique a été équipée d'un dispositif générateur de tourbillons qui sera la source de perturbations aux caractéristiques dynamiques et cinématiques contrôlables.

Le dispositif adopté est constitué d'un volet oscillant autour d'un axe horizontal perpendiculaire à l'axe longitudinal de la soufflerie (Fig. 2). Le volet a une corde de $10 \mathrm{~cm}$ de long et une longueur de $64 \mathrm{~cm}$ afin de traverser la veine d'air. Il est situé sur la "plage " amont à $15 \mathrm{~cm}$ du pont de raccordement entre l'eau et la plage. Il oscille entre la position horizontale et un angle de $30^{\circ}$.

La vitesse de l'air, $U$, a été fixée à des valeurs comprises entre 0,6 et $2 \mathrm{~m} / \mathrm{s}$. Compte tenu des valeurs de $U$, la fréquence d'oscillation du volet permettant d'obtenir des tourbillons énergétiques, stables et pouvant satisfaire la condition de résonance, a été fixée à $2 \mathrm{~Hz}$.

\subsection{Les techniques de mesure}

Le niveau de l'interface a été mesuré à l'aide de sondes capacitives constituées chacune d'un fil conducteur isolé de diamètre extérieur $0,3 \mathrm{~mm}$. Chaque sonde est intégrée dans un circuit électronique (DISA 51 E 02) qui délivre une tension analogique proportionnelle à la longueur de sonde immergée.

Les fluctuations de vitesses dans l'écoulement d'air sont mesurées au moyen d'anémomètres à fils chauds à 


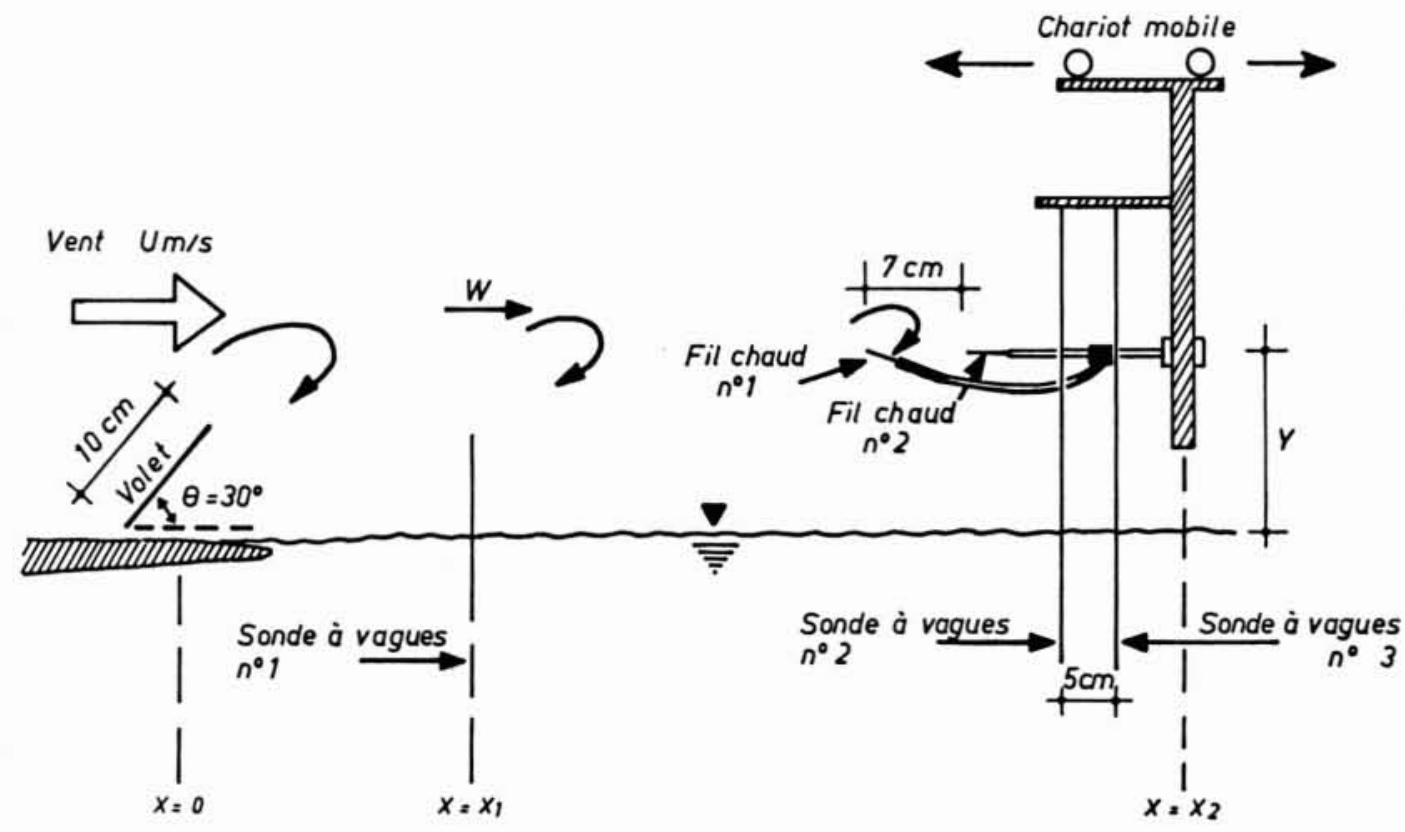

Figure 2 - Dispositif expérimental.

résistance constante. Chaque fil est couplé à une chaîne anémométrique (DISA $55 \mathrm{M} 01$ ) qui fournit une tension $E$ liée à la vitesse $V$ par la relation de type loi de King :

$$
E^{2}=A+B V^{n}
$$

Les tarages ont été effectuée dans une soufflerie d'étalonnage. Les coefficients de la loi (14) ont été calculés par approximation des moindres carrés (Giovanangeli, 1981).

Au cours des expériences, les sondes sont placées sur un support mobile pouvant se déplacer verticalement et longitudinalement dans l'axe de la soufflerie afin de déterminer les vitesses dans l'air $u(t)$ et les dénivellations $\eta(t)$ en fonction du fetch $X$ (Fig. 2).

\subsection{Traitement des données}

Les tensions analogiques fournies par les différentes chaînes de mesure ont été numérisées et traitées à l'aide du calculateur Hewlett-Packard de I'I.M.S.T. à Luminy. La fréquence d'échantillonnage était égale à $40 \mathrm{~Hz}$. Les signaux analogiques ont été au préalable filtrés en passebas à $20 \mathrm{~Hz}$ afin d'éviter le phénomène d'aliasing.

Après numérisation les données ont été stockées sur bandes magnétiques, transformées en paramètres physiques (hauteur de l'interface, vitesse de l'air) à l'aide des lois d'étalonnage et conservées à nouveau sur bandes magnétiques.

La technique de transformée de Fourier rapide (F.F.T.) a été utilisée afin d'estimer les propriétés statistiques telles que spectres et spectres croisés. L'estimation de la célérité de phase $C(n)$ a été obtenue à l'aide de la formule classique :

$$
C(n)=\frac{2 \pi \cdot \Delta x \cdot n}{\Phi(n)}
$$

où $n$ est la fréquence, $\Delta x$, la distance entre deux sondes et $\Phi(n)$, le déphasage entre deux paramètres déterminés par les deux sondes.

La majeure partie des expériences ont été effectuées dans des conditions de vent stationnaire justifiant l'utilisation de l'analyse harmonique par F.F.T. Cependant quelques expériences ont été réalisées par un vent variant continûment au cours du temps. Dans ces conditions, il n'a pas été possible d'utiliser les techniques classiques citées plus haut.

Une technique originale a été introduite (Memponteil, 1983) afin d'estimer les propriétés statistiques du champ de vagues et de fluctuations de vitesse dans l'air pour de telles conditions d'observation. Il a été possible de déterminer sur des intervalles de temps très courts $(3.2 \mathrm{sec})$ les variances et les fonctions de densités spectrales et cospectrales entre les différents paramètres.

\section{Résultats d'expérience}

\subsection{Les perturbations de l'écoulement d'air}

La figure 3 montre, à $X_{1}=1.565 \mathrm{~m}$, et pour une vitesse $U=0.83 \mathrm{~m} / \mathrm{s}$ à l'amont du volet oscillant, un exemple type de variation temporelle de la composante longitudinale, $u$, de la vitesse de l'écoulement d'air et la densité 

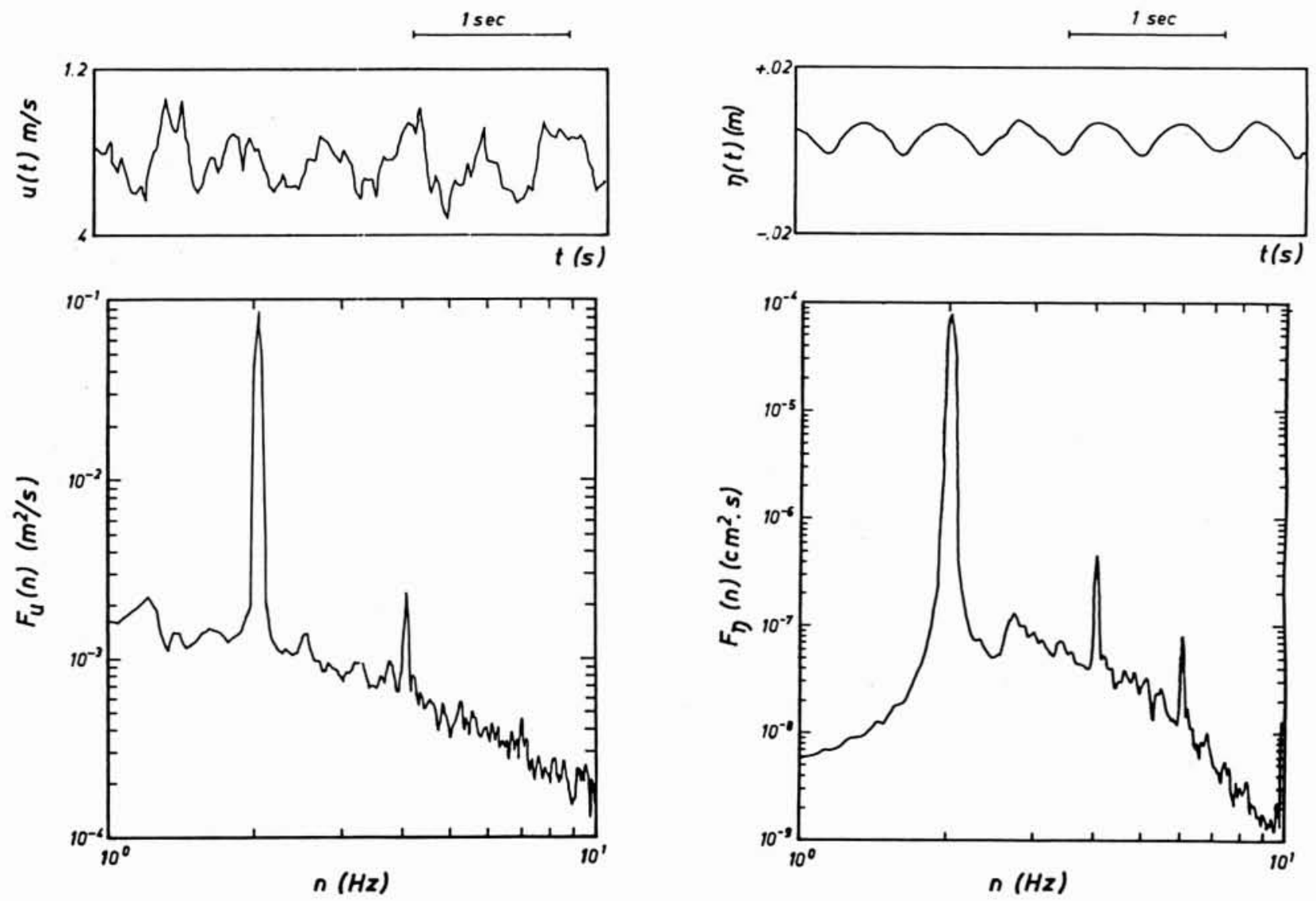

Figure 3 - Variation temporelle de la vitesse et densité spectrale d'énergie correspondante pour $U=0.83 \mathrm{~m} / \mathrm{s}$.

Figure 5 - Variation temporelle du niveau $\eta(t)$ de l'interface pour $U=0.83 \mathrm{~m} / \mathrm{s}$ et densité spectrale correspondante.

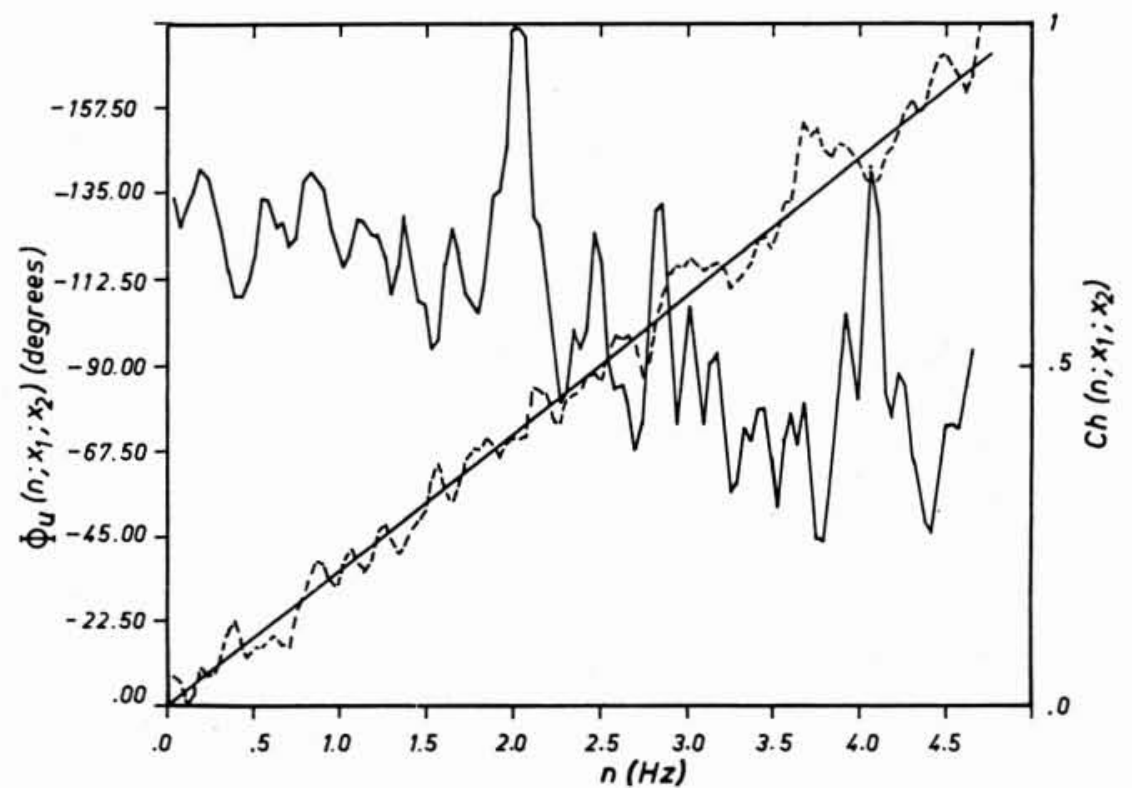

Figure 4 - Fonction de cohérence $C_{h}$ et phase $\Phi_{u}$ entre les fluctuations de vitesse déterminée à $X_{1}=0.57 \mathrm{~m}$ et à $X_{2}=0.64 \mathrm{~m}$. - phase $\Phi_{u}\left(n ; X_{1} ; X_{2}\right) ; \ldots$. cohérence $C_{h}\left(n ; X_{1} ; X_{2}\right)$. 


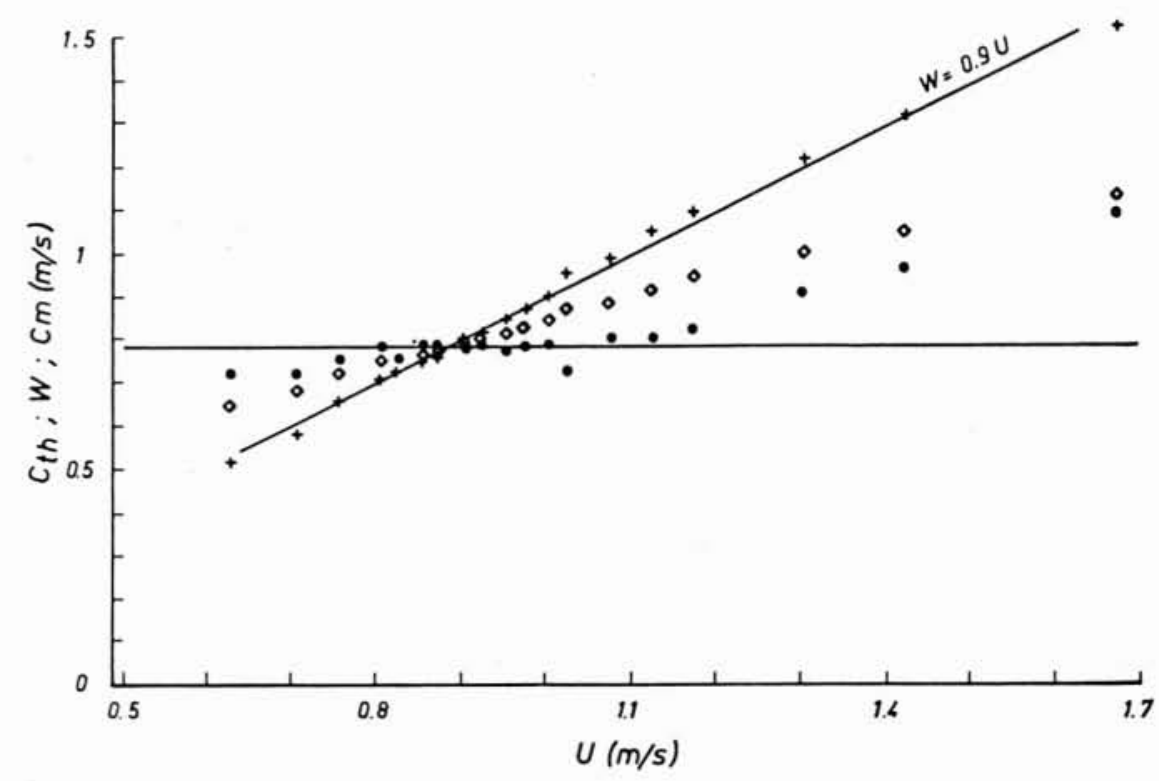

Figure 6 - Célérité de phase des vagues et vitesse de convection des tourbillons pour différentes valeurs de $U$. - Célérité de phase théorique $C_{m}$ :

-+- Vitesse de convection mesurée $W$ des vortex; $\bullet$ Célérité de phase mesurée $C_{m}$ des vagues; $\diamond 1 / 2\left(W+C_{m}\right)$.

spectrale, $F_{u}$, qui lui correspond. On voit clairement apparaître des variations quasi-périodiques à la fréquence d'oscillation du volet $(2 \mathrm{~Hz})$. La forme des fluctuations est en accord avec celles qui doivent être observées au passage de vortex (Sedov, 1973). Le spectre d'énergie présente deux pics principaux à $2 \mathrm{~Hz}$ et $4 \mathrm{~Hz}$.

La fonction de cohérence, $\mathrm{Ch}(n)$, et la phase, $\Phi(n)$, entre les fluctuations de vitesse déterminées à $X_{1}$ et $X_{1}+$ $\Delta X_{1}\left(\Delta X_{1}=7 \mathrm{cms}\right)$ sont présentées sur la figure 4 . La fonction de cohérence présente, comme le spectre, deux pics bien définis à $2 \mathrm{~Hz}$ et à $4 \mathrm{~Hz}$. A $2 \mathrm{~Hz}$, la valeur de la cohérence est pratiquement égale à l'unité.

La phase entre les fluctuations de vitesse déterminées à $X_{1}$ et $X_{1}+\Delta X_{1}$ est pratiquement proportionnelle à la fréquence $n$. A première vue, ceci suggère que le volet oscillant génère des perturbations dans l'air qui sont convectées en aval sans déformation. La vitesse de convection, $W$, des perturbations a été déterminée selon la formule (15), à la fréquence de $2 \mathrm{~Hz}$ qui correspond au maximum de cohérence

Une exploration systématique de l'écoulement d'air a été effectuée pour différentes valeurs de $U$ comprises entre $0.6 \mathrm{~m} / \mathrm{s}$ et $1.7 \mathrm{~m} / \mathrm{s}$. Les résultats ont montré que la variance $\overline{u^{\prime 2}}$ des fluctuations de vitesse dans l'air décroît entre $X_{1}=0.50 \mathrm{~m}$ et $X_{2}=1.25 \mathrm{~m}$ avec un facteur d'amortissement indépendant de la valeur de $U$ et égal à 0.3 . Excepté cet amortissement entre $X_{1}$ et $X_{2}$, la plupart des résultats obtenus à $X_{1}$ restent valables à $X_{2}$. En outre, la vitesse de convection $W$ des tourbillons est pratiquement proportionnelle à la vitesse $U$.

\subsection{Les mouvements de l'interface}

La figure 5 montre, pour $U=0.83 \mathrm{~m} / \mathrm{s}$, la variation temporelle du niveau instantané $\eta(t)$ de l'interface et le spectre d'énergie $F_{\eta}$ correspondant. Comme pour les fluctuations de vitesse, les variations de $\eta$ sont quasipériodiques et le spectre d'énergie présente deux pics principaux à $2 \mathrm{~Hz}$, fréquence du volet, et à $4 \mathrm{~Hz}$. Ces résultats sont valables pour toutes les valeurs de $U$ dans la gamme $0.86 \mathrm{~m} / \mathrm{s}-1.7 \mathrm{~m} / \mathrm{s}$. Ils suggèrent qu'il existe un couplage très net entre les perturbations dans l'écoulement d'air et les mouvements de l'interface.

La célérité de phase des ondes de surface a été déterminée au fetch $X_{2}$ en utilisant la formule (15). Les résultats montrent (Fig. 6) que, dans la gamme $0.8 \mathrm{~m} / \mathrm{s}<U<1.1 \mathrm{~m} / \mathrm{s}$, la célérité de phase mesurée $C_{m}$ est proche de $C_{T h}$, valeur prévue par la théorie des ondes de gravité. Pour $U=0.86 \mathrm{~m} / \mathrm{s}$, on trouve que $C_{m}=C_{m}$ $=W$.

Le champ de vagues est alors principalement constitué d'une onde libre $\left(C_{m}=C_{T h}\right)$ résonnante $\left(C_{m}=W\right)$. Pour les autres gammes de vitesse, on constate que la valeur de la célérité mesurée $C_{m}$ croît lorsque $U$ augmente.

La figure 7 présente les valeurs des variances $\overline{\eta^{2}\left(X_{1}\right)}$ et $\overline{\eta^{2}\left(X_{2}\right)}$ des fluctuations du niveau de l'interface à $X_{1}$ et $X_{2}$ en fonction de "l'âge " de la vague $C_{m} / W$ et le coefficient d'amplification

$$
\alpha=\frac{\overline{\eta^{2}\left(X_{2}\right)}-\overline{\eta^{2}\left(X_{1}\right)}}{\overline{\eta^{2}\left(X_{1}\right)}}
$$


Il apparaît clairement sur cette figure que $\alpha$ présente un maximum bien défini pour $C_{m} / W$ proche de l'unité. Les évolutions de $\overline{\eta^{2}\left(X_{2}\right)}$ et de $\overline{\eta^{2}\left(X_{1}\right)}$ montrent que ce maximum correspond bien à un maximum d'amplification du champ de vagues entre $X_{1}$ et $X_{2}$. Ces résultats suggèrent bien la validité d'un processus de résonance.

Le caractère sélectif de la réponse de l'interface est confirmé sur la figure 8 qui donne l'évolution, en fonction du fetch, du champ de vagues pour $C_{m} / W=1$ et $C_{m} / W$ $=0.9$. L'énergie des vagues est rapportée à l'énergie des fluctuations de vitesse dans l'air. On constate qu'à partir de $X=100 \mathrm{~cm}$ les deux évolutions sont différentes : pour $C_{m} / W=1$, l'énergie des vagues continue à augmenter et se stabilise, alors que pour $C_{m} / W=0.9$, l'énergie décroît rapidement. La stabilisation de l'énergie des vagues pour $C_{m} / W=1$ pourrait s'expliquer par l'amortissement en énergie que subissent les vortex aériens au cours de leur propagation.

Le second cas d'observation ( $C_{m} / W=0.9$ ) correspond à la condition non résonnante qui conduit effectivement à une modulation dans l'amplitude des vagues. A partir des modèles analytiques et numériques on peut montrer que la longueur d'onde de modulation dans l'espace de l'amplitude des vagues est :

$$
X=\frac{L \cdot C g}{|W-C|}
$$

où $C g=C / 2$ est la vitesse de groupe de l'onde non résonnante. Pour le cas d'observation considéré ici $\left(C_{m} / W\right.$ $\left.=0.9, W=0.865 \mathrm{~m} / \mathrm{s} ; C_{m}=0,776 \mathrm{~m} / \mathrm{s}, L=0.43 \mathrm{~m}\right)$, on trouve $X=1.87 \mathrm{~m}$ valeur compatible avec celle qui peut être estimée sur la figure 8 en considérant le début de l'augmentation de l'énergie des vagues à $X \simeq 0.6 \mathrm{~m}$.

\section{Discussions}

Les résultats présentés ici ont mis en évidence le rôle important du mécanisme de résonance dans la génération des vagues à l'interface air-eau sous l'action d'une allée de tourbillons. En outre, ils ont montré l'existence d'un système de vagues non résonnantes qui pourrait jouer un rôle important dans le développement des vagues de vent.

Afin de vérifier si le méchanisme de génération par résonance pouvait être efficace dans le cas où le vent n'est plus constant mais varie au cours du temps, une expérience a été mise en œuvre à l'aide du même dispositif expérimental. La vitesse du vent $U$ a varié de 0 à $3 \mathrm{~m} / \mathrm{s}$ en $20 \mathrm{sec}$. Les propriétés statistiques des champs de vagues et des fluctuations de l'écoulement d'air ont été estimées sur des échantillons de $3.2 \mathrm{~s}$ à l'aide d'une méthode basée sur l'estimation spectrale par maximum d'entropie (voir par exemple Memponteil, 1983).

La figure 9 montre que, comme pour le cas stationnaire, le processus de résonance reste encore efficace et que la réponse de l'interface est pratiquement instantanée.

Les modèles théoriques et numériques ont montré que dans le cas non résonnant le champ de vagues pouvait être la superposition d'ondes libres et d'ondes forcées. Les résultats expérimentaux tendent à confirmer une telle hypothèse, sans pour autant nous permettre de séparer les ondes libres des ondes liées à cause des méthodes statistiques utilisées.

Afin de mettre en évidence la nature exacte des mouvements de l'interface, une procédure simple a été utilisée : le niveau instantané de l'interface a été enregistré au cours du temps, successivement pour différents fetchs distants de $2 \mathrm{~cm}$.

Les enregistrements sont illustrés sur les figures 10 et $10 \mathrm{bis}$ pour $C_{m} / W=0.73(W=1.25 \mathrm{~m} / \mathrm{s})$. On constate que pour un fetch donné, le mouvement de l'interface est constitué d'une oscillation de grande amplitude sur laquelle se superpose une onde d'amplitude nettement plus petite indiquée par une flèche. On remarque aussi que le déphasage entre ces deux composantes évolue avec le fetch. Les deux composantes semblent donc se propager à des célérités de phase différentes que nous noterons $C_{D}$ et $C_{R}$ respectivement pour les composantes de grande et de petite amplitude.

La valeur de $C_{D}$ a été déterminée directement à partir d'enregistrements sur papier effectués à $X$ et $X+\Delta X$ $(\Delta X=5 \mathrm{~cm})$. Il a été trouvé la valeur $C_{D}=1.04 \mathrm{~m} / \mathrm{s}$. Cette méthode n'a pu être mise en œuvre pour la deuxième composante à cause de la faible valeur de l'amplitude de cette composante. Une autre méthode a été employée.

En effet, soit $k_{R}$ et $k_{D}$ les nombres d'ondes correspondants à la petite et la grande oscillation, et soit $\Delta X$ la distance requise pour obtenir un cycle complet dans l'évolution de leur phase respective, on a alors

$$
2 \pi=\left|k_{R}-k_{D}\right| \Delta X
$$

soit :

$$
C_{R}=\frac{C_{D} \cdot n \cdot \Delta X}{C_{D}+n \Delta X}
$$

$n$ est la fréquence considérée (ici $2 \mathrm{~Hz}$ ); $\Delta X \simeq 0.24 \mathrm{~m}$; $C_{R}$ est de l'ordre de $0.32 \mathrm{~m} / \mathrm{s}$.

Les valeurs de $C_{D}$ et $C_{R}$ trouvées à partir de l'expérience sont proches de celles données par les modèles, à savoir :

$$
C_{D}=\frac{1}{2}\left(W+C_{T h}\right)=\frac{1}{2}(1.25+0.78)=1.02 \mathrm{~m} / \mathrm{s},
$$

et

$$
C_{R}=\frac{1}{2}\left(W-C_{T h}\right)=0.24 \mathrm{~m} / \mathrm{s} .
$$

Ces résultats suggèrent très fortement que, lorsque la condition de résonance n'est pas satisfaite, les trois composantes du champ de vagues (onde libre se propageant à $+C$, onde libre se propageant à $-C$, onde liée se propageant à $W$ ) se combinent entre elles pour donner deux ondes se propageant à $\left(W+C_{T_{h}}\right) / 2$ et $\left(W-C_{T_{h}}\right) / 2$, célérités dépendant directement de la vitesse du vent $W$.

On a vu également qu'un champ de vagues pouvait être pratiquement instantanément généré par un champ de perturbations dans l'écoulement d'air par l'intermédiaire d'un mécanisme de résonance. Hors résonance, le champ de vagues apparaît comme la superposition d'ondes " directes" ou "rétrogrades " pouvant se propager dans le sens du vent ou même en sens contraire. Ces ondes non résonnantes peuvent jouer un rôle déterminant dans l'évolution de nombreux champs de vagues incluant notamment celui des vagues de vent. 


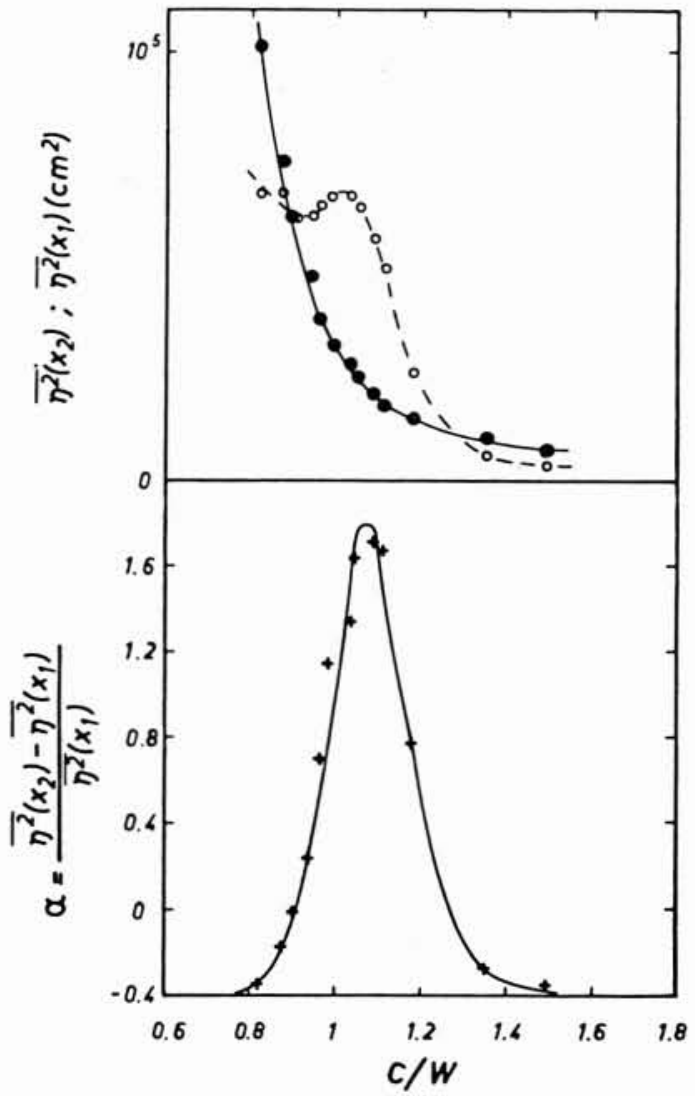

Figure 7 - Courbe supérieure : Energie des vagues en fonction de l'âge $C_{m} / W$ des vagues pour $X=X_{1}(-\cdot-)$ et pour $X=X_{2}$ $(-0-)$. Courbe inférieure: Coefficient d'amplification $\alpha$ de T'énergie des vagues entre $X_{1}$ et $X_{2}$ en fonction de $C_{m} / W$.

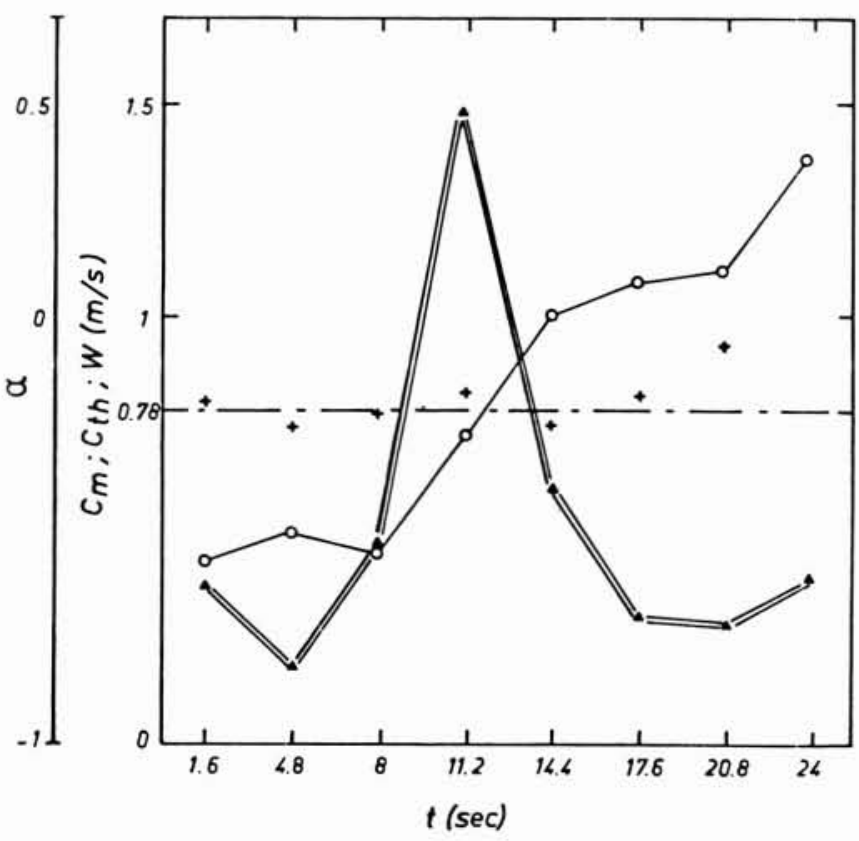

Figure 9 - Coefficient d'amplification $\alpha$, célérité de phase théorique $C_{m}$ et célérité de phase mesurée des vagues $C_{m}$; vitesse de convection des tourbillons en fonction du temps pour une condition expérimentale non stationnaire :

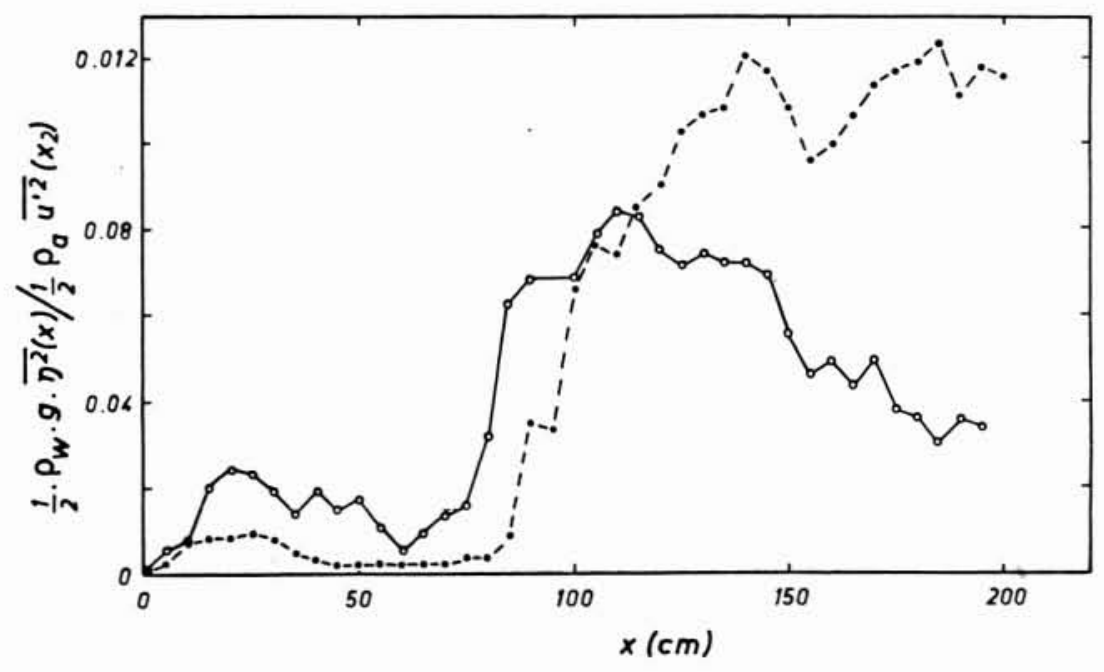

Figure 8 - Evolution avec le fetch de l'énergie des vagues. $-\cdot-$ pour $C_{m} / W=1 ;-0-$ pour $C_{m} / W=0.9$. 

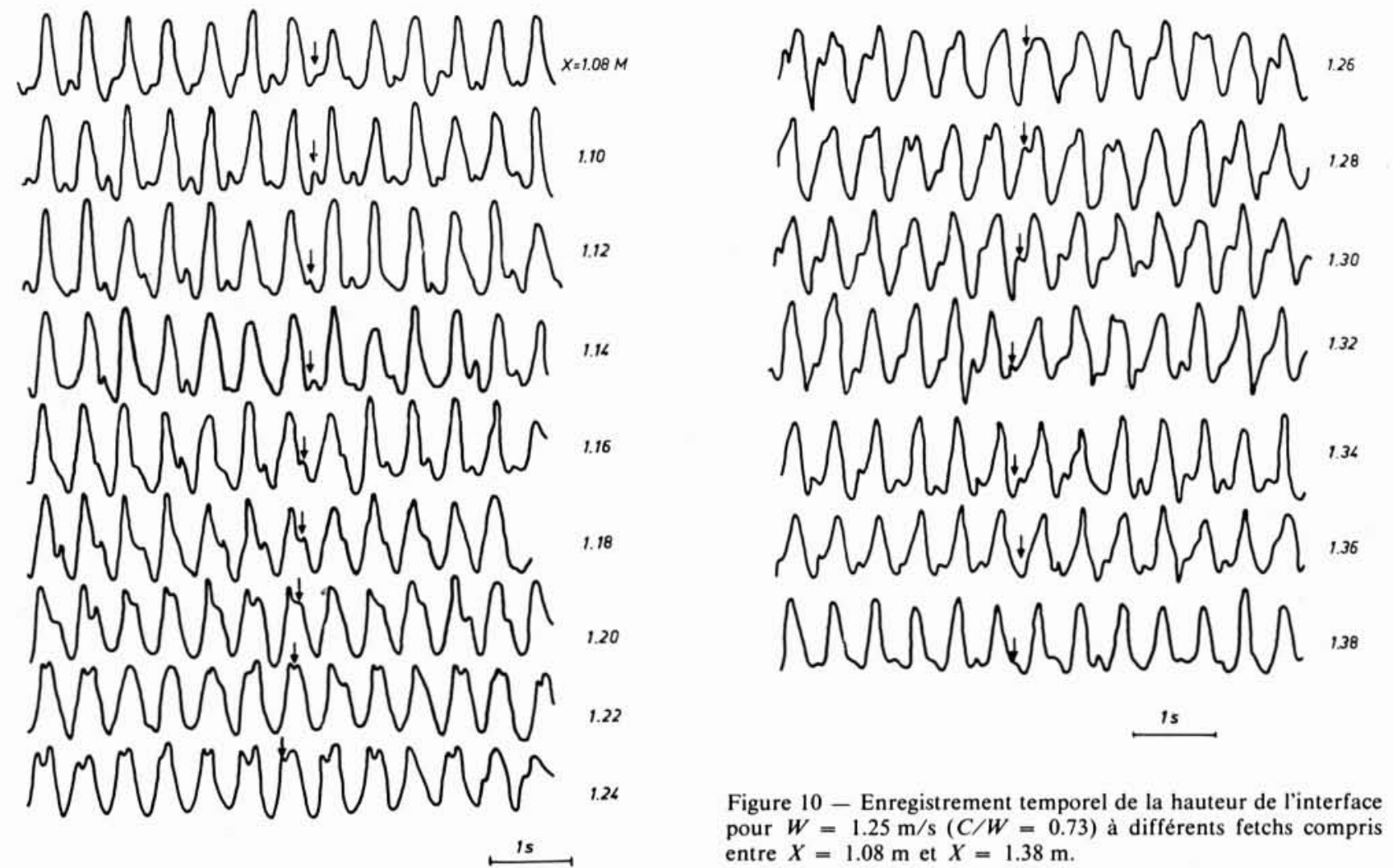

\section{Références}

Сно1 1. (1977). - Contribution à l'étude des mécanismes physiques de la génération des ondes de capillarité gravité à une interface air-eau. Thèse de $3^{\circ}$ Cycle - I.M.S.T.

Favre A. et Coantic M. (1974). - Activities in, and preliminary results of, air-sea interactions research at I.M.S.T. $A d v$. Geophys. 18A.

GELCI R. (1974). - Calcul numérique de certains écoulements par la théorie des tourbillons. La Houille Blanche $\mathrm{n}^{\circ} 6$.

Gelci R. et Hervouet J.Y. (1981). Traitement numérique d'une surface libre. Méthodes des singularités Lagrangiennes. Rapport d'avancement, Centre de Météorologie Maritime de l'E.E.R.M.

Gelci R., Ramamonjiarisoa A. et Hervouet- J.Y. (1983). Génération de vagues de gravité par des allées de tourbillons aériens mobiles. Note E.E.R.M. $\mathrm{n}^{\circ} 54$.

GelCl R. et Hervouet J.Y. (1983). Ondes de pression générant des ondes de gravité. Note E.E.R.M. $\mathrm{n}^{\circ} 72$.

Giovanangeli J.P., Revault D'Allonnes M. et RamamoniariSOA A. (1978). - Open sea simultaneous observations of air and water motions during active air-sea interactions. Proc. "Turbulent Fluxes through the Sea Surface Waves Dynamics and Prediction ", Plenum Press.

Giovanangel J.P. (1980). - A non dimensional heat transfer law for a slanted hot film in water flow. DISA Info. $\mathrm{n}^{\circ} 25$.
Giovanangeli J.P. et MemponteIl A. (1983), - An experimental study of resonant and non resonant wind waves. A paraitre. HASSELMANN K. (1962), - On the non-linear energy transfer in a gravity wave spectrum. Part 1., J. Fluid Mech., 12, 481-500.

LONGUET-Higgins M.S. (1962). Resonant interactions between two trains of gravity waves. J. Fluid Mech., 12, pp. 321-332.

Longuet-Higgins M.S. et Phillips O.M. (1962). - Phase velocity effects in tertiary wave interactions. J. Fluid Mech., 25, 417-435.

MemponteIl A. (1983). - Réponse d'une surface d'eau à des mouvements tourbillonnaires dans l'écoulement d'air adjacent. Thèse de $3^{\circ}$ Cycle, Université d'Aix-Marseille II;

MiLES J.W. (1960). - On the generation of surface waves by turbulent shear flow. J. Fluid Mech., 7, part 3.

Mollo-Christensen E. et Ramamonjiarisoa A. (1978). Modeling the presence of waves groups in a random wave field. J. Geophys. Res., 83.

PHILLIPS O.M. (1957). - On the generation of waves by turbulent wind. J. Fluid Mech., vol. 2, part 5.

PHILLipS O.M. (1966). - Dynamic of the upper ocean. Cambridge Univ. Press.

Sedov L. (1973). - Mécanique des milieux continus. M.I.R.

StOKER J.J. (1957). - Water waves. Intersciences Publ. Inc., N.Y. 